\title{
Existence Theorems and Measurement of the Capillary Contact Angle
}

\author{
B.S. FISCHER and R. FINN
}

\begin{abstract}
In an earlier work, Concus and Finn proposed two procedures, based on a dichotomous behavior of fluid in capillary tubes at zero gravity, designed for accurate measurement of capillary contact angles. The present work offers modifications to one of their procedures which should facilitate the experiments and lead to improved accuracy. The discussion leads to explicit characterization of a capillary tube with "canonical" section, which has an independent mathematical interest.
\end{abstract}

Key words: Capillarity, contact angle, dichotomous behavior, canonical proboscis AMS subject classification: $76 \mathrm{~B} 45,53 \mathrm{~A} 10,49 \mathrm{Q} 10$

\section{Background remarks}

This paper can be regarded as a continuation of the discussion relating to the "second method" in the earlier. work by Concus and Finn [4]. The "second method" was proposed by those authors as a procedure for obtaining accurate measurements of the fluid-solid-vapor capillary contact angle $\gamma$, when $\gamma$ is close to zero; it is based on height measurements, near the boundary arc of smaller radius, of the free surface interface in cylindrical capillary tubes whose sections are bounded by two circular arcs, as indicated in Figure 1.

The underlying idea in the procedure rests on the theorem that there is a critical angle $\gamma_{0}$, depending only on the geometrical parameters, such that if $\gamma>\gamma_{0}$ then there will exist in equilibrium a connected volume of fluid covering the base $\Omega$ and with bounded height, while if $\gamma<\gamma_{0}$ no such configuration can exist. In the latter case, a formal solution of the variational problem for a (non-parametric) free surface interface of stationary mechanical energy exists only as a "generalized solution" in the sense of Miranda [8], and has height identically equal to infinity on a set of the form of the shaded region $\Omega^{*}$ of Figure 1. Physically, for any given volume $V$ of fluid in the container, as $\gamma$ decreases to $\gamma_{0}$, the fluid height of the equilibrium configuration increases unboundedly over $\Omega^{*}$ until sufficient volume will lie over that domain so that part of the base in $\Omega \mid \Omega^{*}$ will become uncovered: The height changes necessarily become very rapid when $\gamma$ is close to $\gamma_{0}$.

In principle, this "near-discontinuous" behavior should lead to a very easy way to determine $\gamma_{0}$, in which hysteresis effects would be overcome by much larger capillary forces

B. S. Fischer: Massachusetts Int. Techn., Math. Dep., Cambridge, MA 02139, USA

R. Finn: Stanford University, Math. Dep., Stanford, CA 94305, USA 
and in which precise observations near the contact line need not be made; that is, it should suffice to find configurations in which the fluid tends to empty those parts of the container that are not over $\Omega^{*}$. In practice, a difficulty can arise in that $\Omega^{*}$ can have relatively small area and thus the vessel would have to be unrealistically tall to permit the observation. The difficulty can presumably be alleviated to some extent by placing a sponge at the top, so that once the fluid rises an additional negative pressure would be created causing it to flow to the top; nevertheless, it was contemplated in [4] that fluid heights be observed at points near the arc $\Sigma^{*}$; these observations should be facilitated by the circumstance that the "high-rise" occurs along the entire arc $\Sigma^{*}$.

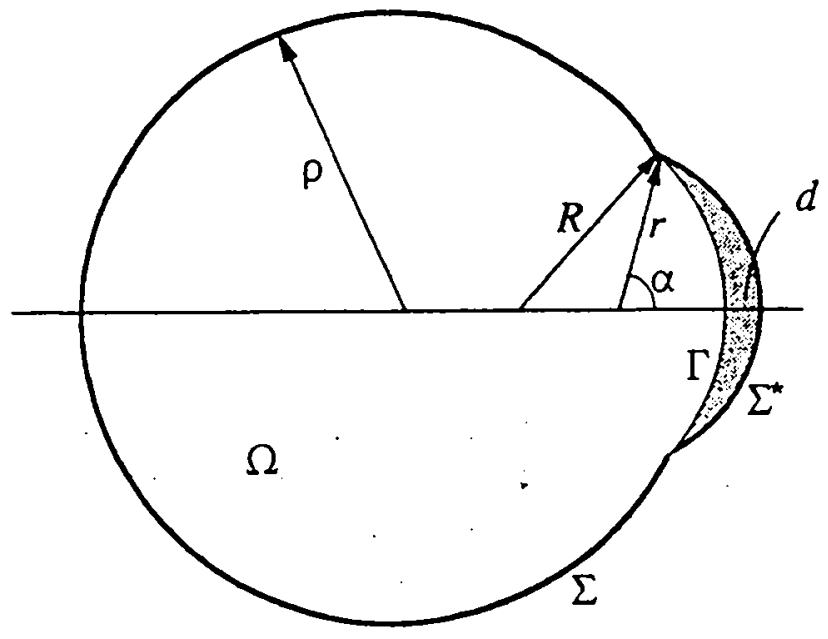

Figure 1. Two circle domain; extremal $\Gamma$, singular region

For the theoretical background of the procedures we shall use, we refer the reader to [6], Chapters 6 and 7, see also [3]. We state here only the formal result that we need. We consider a semi-infinite cylindrical tube $Z$ with section $\Omega$, whose boundary $\sum$ consists of a finite number of smooth arcs meeting at well-defined (interior) angles $2 \phi$. A corner defined by such an intersection point is called reentrant if $2 \phi>\pi$. We seek capillary surfaces $u(x, y)$ defined over $\Omega$, bounding with $\Omega$ a (prescribed) finite volume $V$ of fluid, and meeting the bounding walls over the smooth parts of $\Sigma$ in a prescribed (constant), angle $\gamma$. Any such surface is determined as solution of the equation

in $\Omega$, with

$$
\operatorname{div} T u=2 \ddot{H}
$$


and

$$
T u=\frac{\nabla u}{\sqrt{1+|\nabla u|^{2}}}
$$

$$
2 H=\frac{|\Sigma|}{|\Omega|} \cos \gamma
$$

under the boundary condition

$$
v \cdot T u=\cos \gamma
$$

on $\Sigma$, where $v$ is exterior unit normal on $\Sigma$. The quantity $H$ can be interpreted geometrically as the mean curvarure of the free surface interface determined by $u(x, y)$. In what follows we restrict ourselves throughout to the "wetting" case $0 \leq \gamma<\pi / 2$. The complementary case $\pi / 2$ $<\gamma \leq \pi$ is obtained on replacing $u$ by its negative, while if $\gamma=\pi / 2$ the constants are the only solutions.

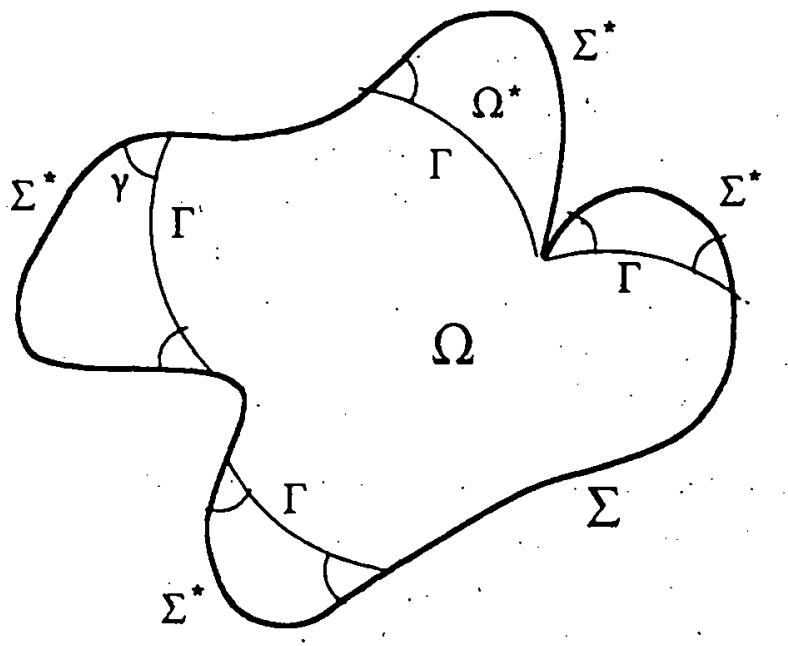

Figure 2. General domain $\Omega ;\{\Gamma ; \gamma\}$ configuration

Definition: A domain $\Omega$ as above will be said to admit a $\{\Gamma ; \gamma \mid$ configurarion if there is a non-null proper subset $\Omega^{*}$, bounded in $\Omega$ by a finite ser $\{\Gamma\}$ of subarcs of semicircles of radius $R_{\varphi}=\frac{1}{2 H}=\frac{|\Omega|}{|\Sigma| \cos \gamma}$, with the three properties:

a) the $\{\Gamma\}$ are disjoint except perhaps at reentrant corner points of $\Sigma$,

b) the curvature vector of each $\Gamma$ is directed exterior to $\Omega^{*}$,

c) each intersection point of any arc $\Gamma \in\{\Gamma\}$ with $\Sigma$ is either a reentrant corner with one sided angle between $\Gamma$ and $\Sigma$ not less than $\gamma$ on the side of $\Gamma$ opposite $t o$ its. 
center and not less than $\pi-\gamma$ on the opposite side, or else a point interior to a smooth subarc of $\Sigma$ where $\Gamma$ and $\Sigma$ meet at angle $\gamma$.

A typical $\{\Gamma ; \gamma\}$ configuration is indicated in Figure 2. follows.

The following result, proved in $|5|$ and in [6] Chapter 6, is basic for the material that Theorem: A solution $u(x, y)$ of (1) - (4) exists for given $\Omega$ and $\gamma \neq 0$ if and only if the functional

$$
\Phi=|\Gamma|-|\Sigma| \cos \gamma+\frac{1}{R_{\gamma}}|\Omega|
$$

is positive for every $\{\Gamma ; \gamma\}$ configuration in $\Omega$. If $\dot{\Phi} \leq 0$ for any one such (non-null) configuration, then there exists one that minimizes. $\Phi$ among all such configurations.

The case $\gamma=0$ requires special consideration and will not be dealt with here.

A solution has physical meaning only when $u(x, y)>0$ in $\Omega$. Following techniques developed in 171 , it is possible to show that under the given hypotheses, every solution is bounded below. Thus, by adding a large enough constant to any particular solution, (equivalent to increasing the volume of fluid sufficiently) it can be converted to a solution with physical meaning. In what follows, we assume that this has been done. We will not distinguish among (physical) solutions corresponding to different values of $V$, as it follows from the general comparison principle (cf. Lemma 4 in [7]) that any two solutions differ by an additive constant and hence are mutually congruent.

The interest of the theorem derives in large part from the circumstance that in many situations there will be only a finite number of arcs $\Gamma$ (aside from trivial rigid displacements arising from symmetries) that satisfy the geometrical conditions; for each such $\Gamma$ (or set $\{\Gamma\}$ ), $\Phi$ can be calculated explicitly. The dichotomous behavior at $\gamma=\gamma_{0}$, as described in the introductory remarks above, is the basis for the proposed measurement procedures. The angle $\gamma_{0}$ is characterized as the greatest lower bound of values $\gamma$ in $[0, \pi / 2\}$ such that $\Phi\left(\Omega ; \Omega^{*} ; \gamma\right)$ is positive for all $\Omega^{*}$ of the type considered. For purposes of the experiment, the domain $\Omega$ is to be chosen so that the subdomain $\Omega_{0}^{*}$ that appears when $\gamma=\gamma_{0}$ is of sufficient size to permit accurate observation. Experience in gravitational situations (cf. [6, p. 119]) and calculations in particular related geometries [1] strongly suggest that as $\gamma \downarrow \gamma_{0}$, the fluid height will not rise rapidly in $\Omega_{0}^{*}$ until $\gamma$ is very close to $\gamma_{0}$, after which it will increase greatly in that domain with small decreases in $\gamma$. Since the surface height must be positive infinite over $\Omega_{0}^{*}$ when $\gamma$ $=\gamma_{0}$, and positive infinite over some domain for any $\gamma<\gamma_{0}$, relatively simple observations should suffice to determine in any given case whether or not $\gamma$ exceeds $\gamma_{0}$. 
The arcs $\Gamma$ appearing in the statement of the theorem are the extremals of a variational problem "subsidiary" to the variational problem (principle of virtual work) giving rise to the original equation and boundary condition, see [6], Chapters 1 and 6.

\section{A proposed modification}

As a possible procedure toward improving accuracy of the two circle geometry introduced in [4] by facilitating observation of the transition behavior at $\gamma=\gamma_{0}$, we propose to extend the bulge by introducing inclined straight segments $L$ as indicated in Figure 3.

The idea here is that by extending the bulge, the thickness $d$ of the rising fluid "sheet" would be correspondingly increased. In fact, that need not occur in general, as the end points of the arc $\Gamma$ separating the "infinite" region from the remainder of $\Omega$ may move down the "channel" formed by the segments $L$. Any such situation can however be modified into another one, with the same $\gamma_{0}$ and with $\Gamma$ contacting at the reentrant points, by changing the radius $\rho$ of the larger circle and the "extension" distance $h$. In fact, this step can be effected in

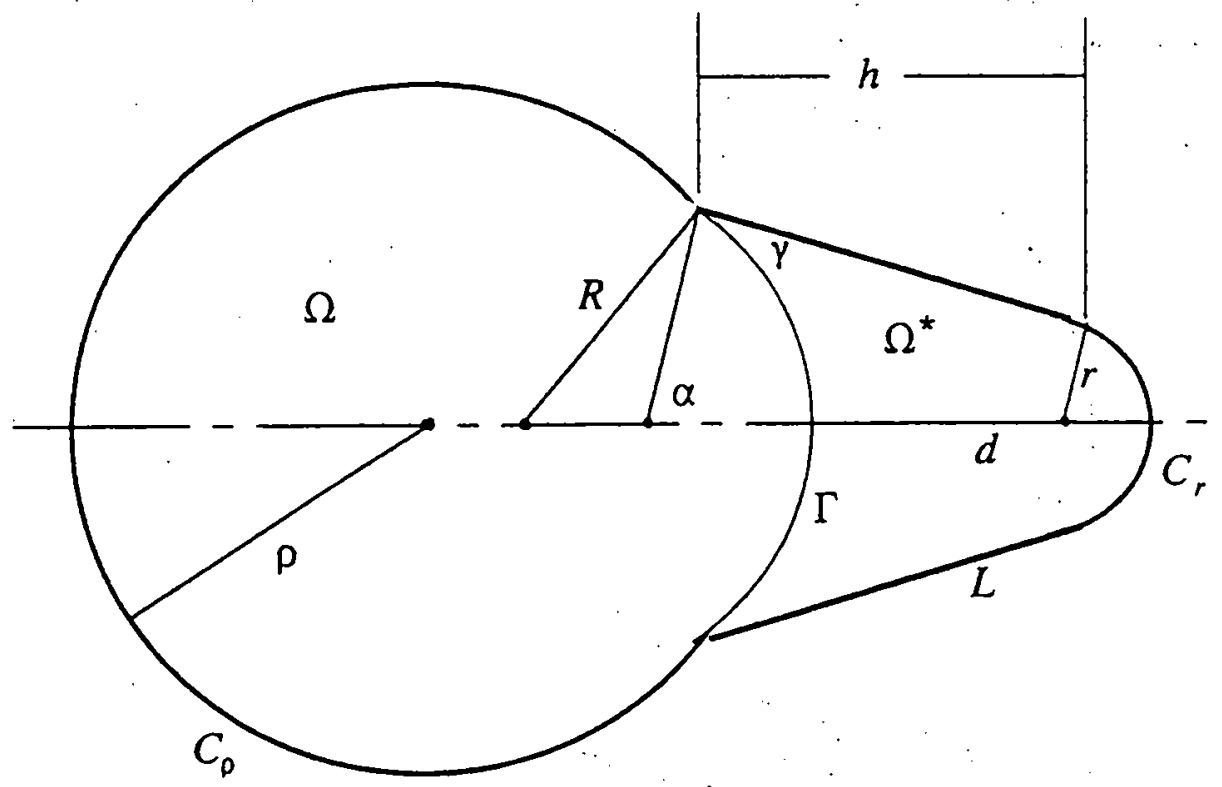

Figure 3. Modified proboscis; extremal $\Gamma$

an infinity of ways, corresponding to incident angles $\gamma^{i}$ of $\Gamma$ with $L$ varying from $\gamma_{0}$ to $\pi / 2$. A heuristic reasoning, supported by the results of our calculations, indicates that the best choice is obtained when $\gamma=\gamma_{0}$, and we restrict attention to that configuration. In general, this step has the effect, for normalized linear dimensions of the cross-section $\Omega$, of leading to a larger 
thickness $d$. For purposes of obtaining the desired configurations, it is simplest to by-pass the intermediate steps and to solve directly for the requisite parameters; we adopt that approach in what follows, and shall study only those configurations that are determined as we have indicated; the actual determination of the parameters corresponding to the indicated criteria was done numerically.

It is clear that the indicated configuration cannot be realized for all data; in particular, $\alpha$ provides an upper bound for the values $\gamma_{0}$ that can be prescribed, and thus the procedure is limited in application to the "small" angles for which it was designed. There was however no difficulty in obtaining numerical solutions for the parameters in suitable ranges, and an existence proof suitable for at least a relevant part of those ranges is not hard to construct. Of much more interest for our context is the question of uniqueness. For the intended purpose of measuring contact angle, it suffices to know that for parameter values $h_{0}, \rho_{0}, r_{0}$ leading to $\Phi_{0}$ $=0$, there can be no $\{\Gamma ; \gamma\}$ configuration, distinct from a single arc $\Gamma_{0}$ situated as the arc $\Gamma$ of Figure 3, which leads to $\Phi \leq 0$; in such a case the fluid could conceivably also rise to infinity in parts of $\Omega$ other than in $\Omega^{*}$. Were such a configuration to exist, there would have to be a minimizing one, by the above Theorem. We consider a particular $\Gamma$ in a minimizing configiration. and examine the cases that can occur.

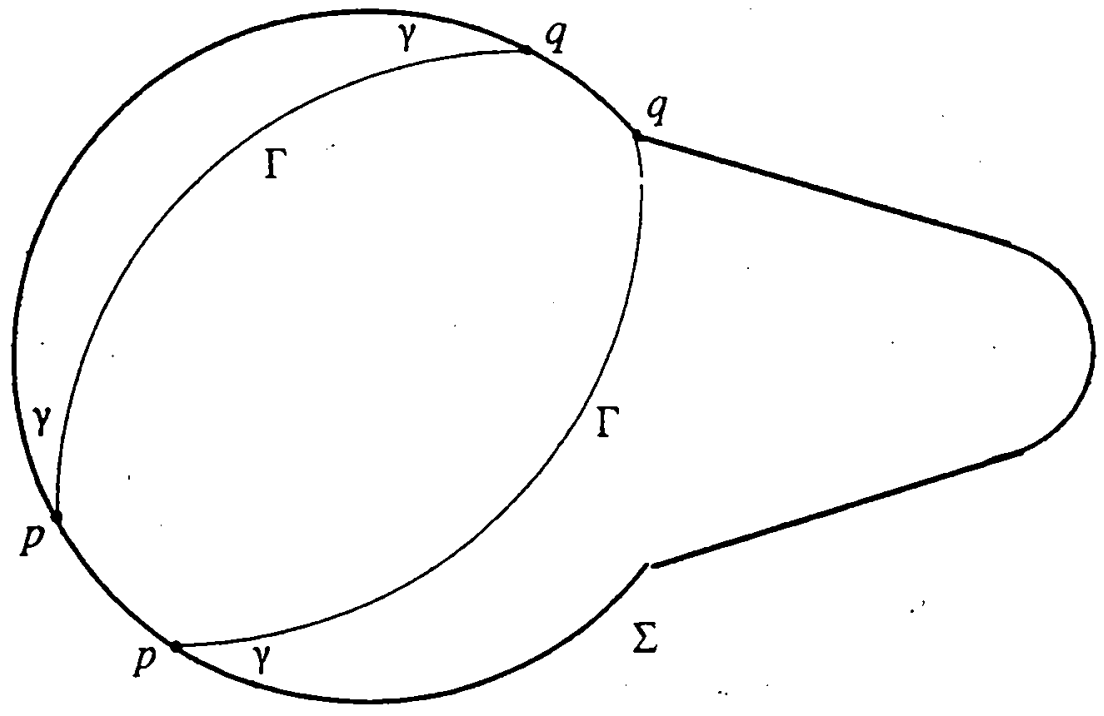

Figure 4. Proof of cases 1 and 2

1. $\Gamma$ meets $C_{\rho_{0}}$ at two interior points $p$ and $q$, as in Figure 4 . This can only occur if $Y_{0}>0$ and $R_{Y_{0}}>\rho_{0}$. Such a configuration would contradict the Corollary to Theorem 6.12 in [6]. 
2. $\Gamma$ meets $C_{\rho_{0}}$ at an interior point $p$ and a reentrant corner $q$, see Figure 4 . A small rotation of $\Gamma$ about the center of $C_{\rho_{0}}$ changes the geometry to the preceding case without changing $\Phi$. We thus obtain a contradiction as in Case 1 .

3. $\Gamma$ meets two interior points, one on each segment of $L$. Then at least one of the angles at the contact points $p$ and $q$ must differ from $\gamma_{0}$, so that $\{\Gamma\}$ cannot minimize.

4. $\Gamma$ meets one point interior to $L$ (or to $C_{r_{0}}$ ) and one reentrant point $p$ on the other $L$ segment. One possibility is the configuration of Figure 5 (or equivalently the reflection of that figure in the symmetry axis). We introduce a parallel to $L$ through $p$ and measure the incident angle $\theta$ with the extremal $\Gamma$, counterclockwise as indicated. By the definition of the $\{\Gamma ; \gamma\}$ configuration we have $2 \alpha-\theta \geq \pi-\gamma_{0}$. Now observe that the indicated configuration can be obtained by rigid (counterclockwise) rotation of $\Gamma$ about $p$, starting with an initial position in which $\Gamma$ is the reflection, in the line joining the two reentrant points, of the arc $\Gamma_{0}$ (Figure 6). Denoting by $\theta_{0}$ the initial value of $\theta$, we have $\theta>\theta_{0}$. By construction, $\theta_{0}=\gamma_{0}$, unless $\alpha$ $=\pi / 2$, in which case $\theta_{0}=\gamma^{i}>\gamma_{0}$. Thus in any case $\theta_{0} \geq \gamma_{0}$, and there follows $2 \alpha+\gamma_{0}-\pi \geq \theta$ $>\theta_{0} \geq \gamma_{0}$, from which $2 \alpha>\pi$, putting $\alpha$ outside the range of parameters contemplated in the construction. Thus this configuration is excluded.

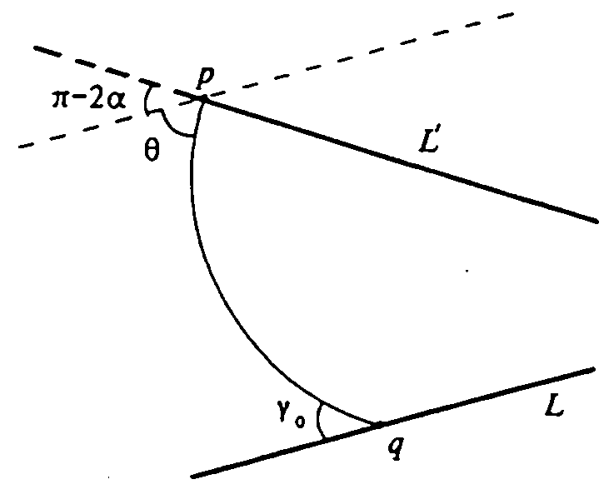

Figure 5. Proof of Case 4

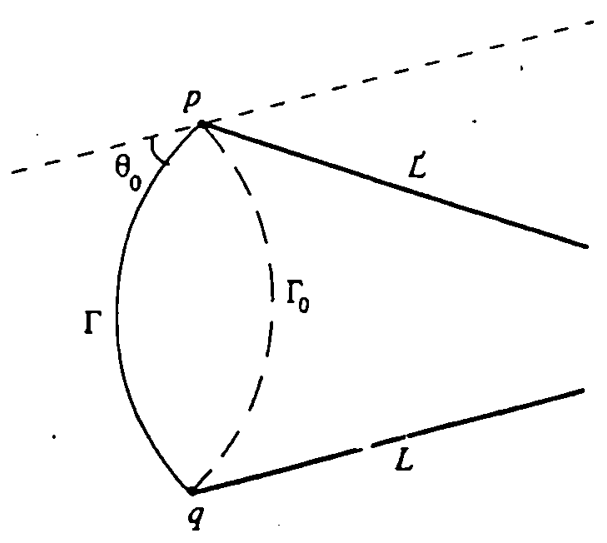

Figure 6. Proof of Case 4; continued

The only other possibility in this category is the identical figure but with the sense of curvature of $\Gamma$ reversed. In this case the incident angle at the reentrant point would be less than $\gamma_{0}$, and thus $\Gamma$ would not be in an admissible configuration.

5. I meets an interior point of $L$ and a point of $C_{r_{0}}$. Then both incident angles must be equal to $y_{0}$. If we extend $L$ as indicated in Figure 7 we obtain a contradiction, as the 
indicated triangle could not be isosceles unless the length of the indicated segment on $L$ were zero.

6. $\Gamma$ meets $C_{r_{0}}$ at two interior points. The reasoning of Case 1 applies.

7. $\Gamma$ meets $\Sigma$ in points $p, q$, with $p \in C_{\rho_{0}}$ and $q \in L$. We distinguish two cases, according to the sense of concavity of $\Gamma$ relative to $L$
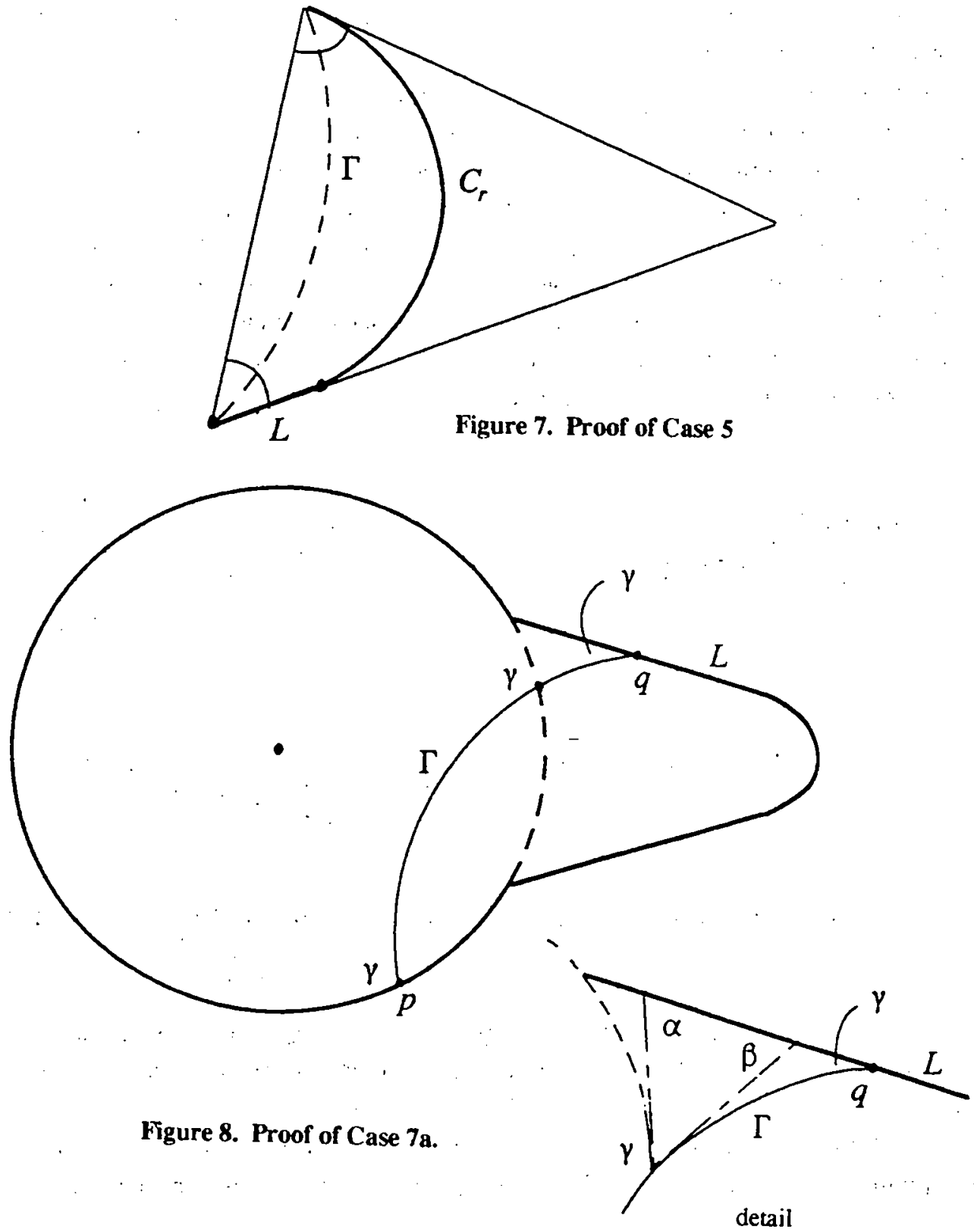
7a) In the configuration of Figure 8 we find $\gamma=\alpha+\beta$ and $\beta>\gamma$. Thus this configuration cannot occur.

7b) We wish to exclude also the reverse concavity of $\Gamma$. We denote by $2 \delta$ the angle subtended by $\Gamma$ between $\rho$ and $q$, and by $2 \delta_{0}$ the portion of that angle within the completed $C_{p_{0}}$. We distinguish two subcases, according as $\delta_{0}+\gamma_{0} \geq \pi /$ or $\delta_{0}+\gamma_{0}<\pi /$. In the former case, $\delta+\gamma_{0}>\delta_{0}+\gamma_{0} \geq \pi /$, and we conclude from the second half of Theorem 6.16 of [6] that $\Gamma$ cannot minimize (see Figure $9 \mathrm{a}$; note that the center of $C_{\rho_{0}}$ has been placed at the origin and the figure has been rotated from the configuration introduced in Figure 3 , so as to fix the center of $\Gamma$ on the $y$-axis).

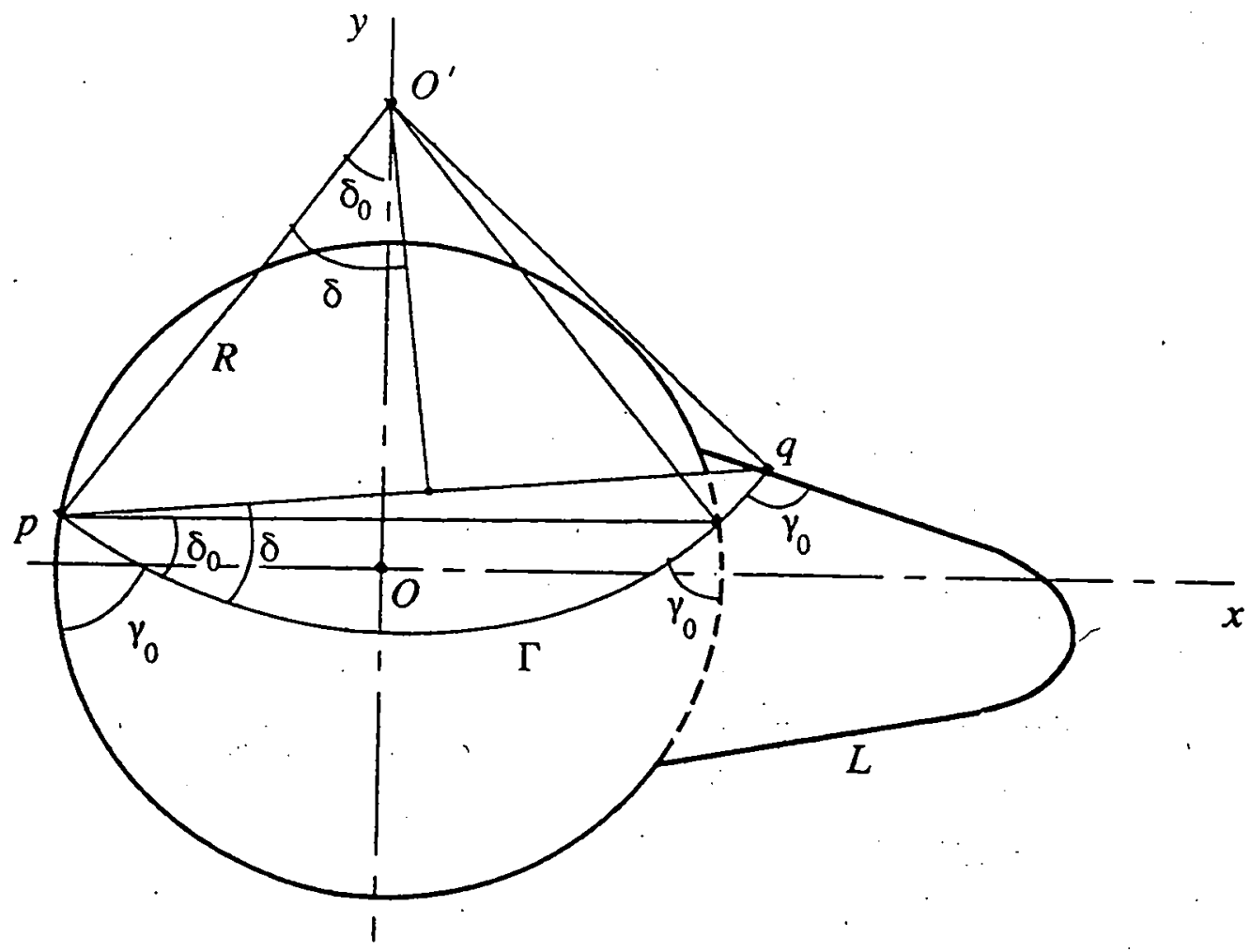

Figure 9a. Proof of case 7b: $\delta_{0}+\gamma_{0} \geq \pi 12$

If $\delta_{0}+\gamma_{0}<\pi / 2$, then necessarily $R \gamma_{0}>\rho_{0}$, and up to rigid rotation about the center $O$ of $C_{p_{0}}$ and reflection in a diameter of $C_{\rho_{0}}$, the configuration must be as in Figure $9 \mathrm{~b}$. We may assume that the intersection $q$ of $L$ with $\Gamma$ lies on or above the $x$-axis; if not, we replace $L$ 
by a line through the intersection of (the extension of) $\Gamma$ with the $x$-axis, making the same angle $\gamma_{0}$ with $\Gamma$, and label this new intersection point with $q$.

Our next step is to replace (the resultant) $L$ with a line $L$, through $q$ and tangent to $C_{\rho_{0}}$. at a point $\left(x_{0}, v_{0}\right)$. Then $y_{0}>0$ and $L_{1}$ will meet $\Gamma$ in an angle $\gamma_{1} \leq \gamma_{0}$ (Figure 9c). We compute formally

$$
\begin{gathered}
\cos \gamma_{0}=\frac{\rho^{2}+R^{2}-b^{2}}{2 \rho R} \\
\cos \gamma_{1}=\frac{\rho^{2}-b y_{0}}{\rho R}
\end{gathered}
$$

so that

$$
\begin{gathered}
2 \rho R\left(\cos \gamma_{0}-\cos \gamma_{1}\right)=R^{2}-\rho^{2}-b^{2}+2 b y_{0} \\
>R^{2}-\rho^{2}-b^{2} .
\end{gathered}
$$

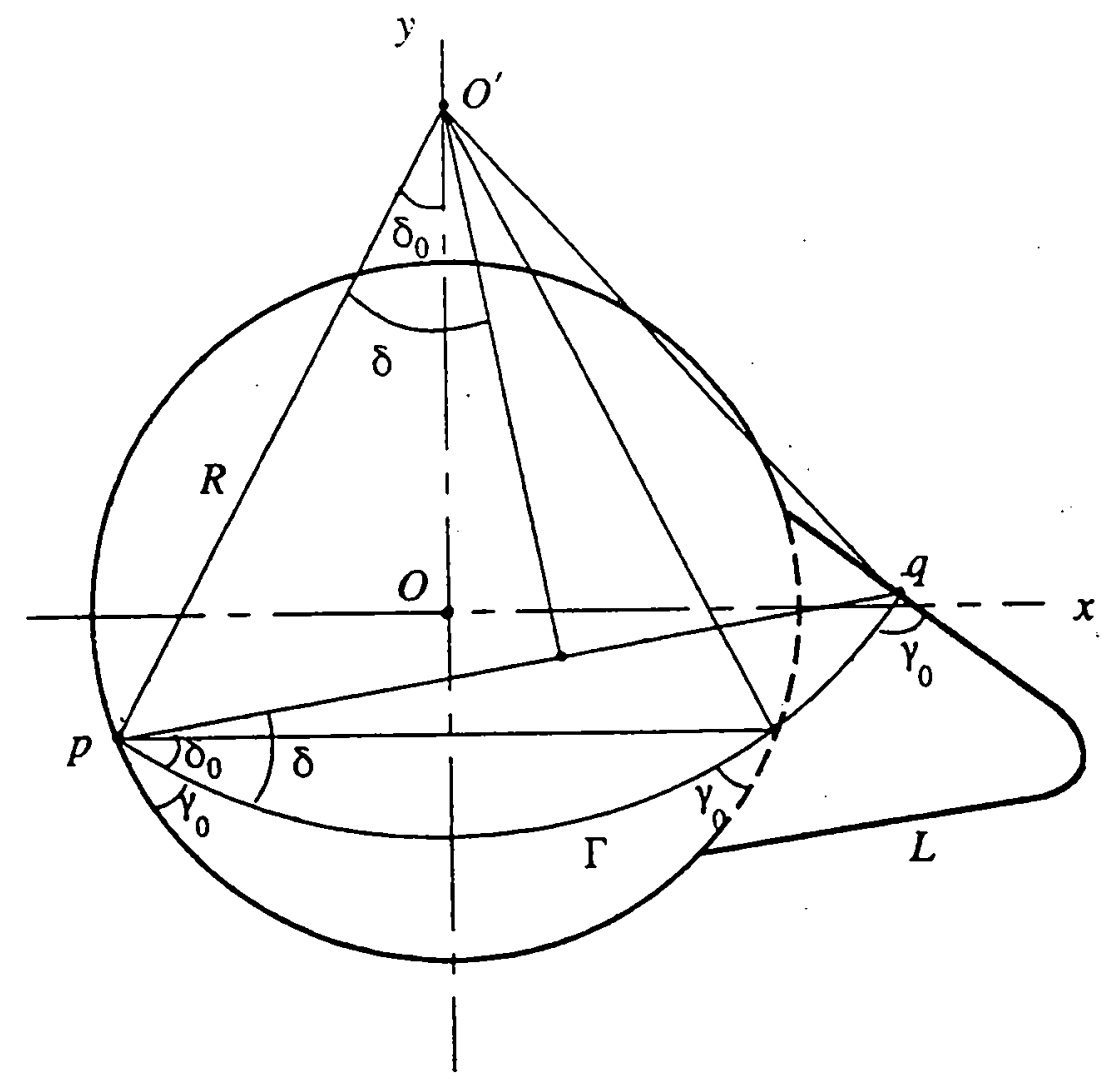

rigure Sh. Proof of case $7 \mathrm{~b}: \delta_{0}+\gamma_{0}<\pi / 2$ 
Since $\delta_{0}+\gamma_{0}<\pi 2, p$ lies below the $\mathrm{x}$-axis. At $p$, we have

from which

$$
\rho^{2}-y^{2}=R^{2}-(b-y)^{2}
$$

$$
-2 b y=R^{2}-\rho^{2}-b^{2}>0
$$

and we see from (8) that $\cos \gamma_{0}>\cos \gamma_{1}$. That is, $\gamma_{1}>\gamma_{0}$, which is not possible since the construction has been effected so that $Y_{0} \geq \gamma_{1}$. Thus, the indicated configuration cannot occur.

8. $\Gamma$ meets $\Sigma$ in points $p \in C_{p_{0}}$ and $q \in C_{r_{0}}$. We divide this case into two subcases:

8a) $R_{\gamma_{0}} \geq \rho_{0}$. Then $R_{y_{0}}\left(\frac{1}{\rho}+\frac{1}{r}\right)>2 \cos \gamma$, contradicting the Corollary to Theorem 6.12 of [6].

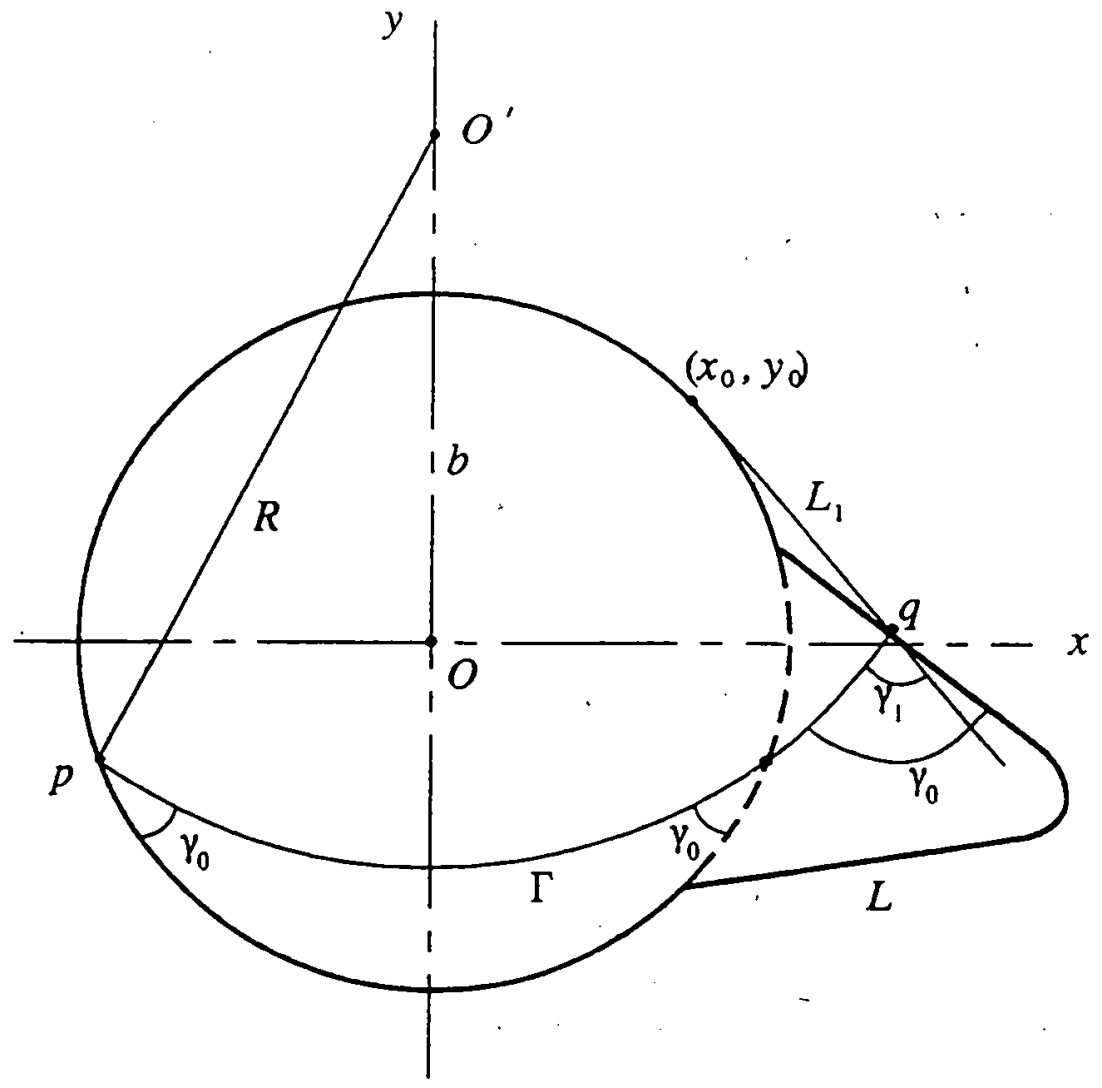

Figure 9c. Proof of case 7: completion of case $7 \mathrm{~b}$ 
8b) $R_{\gamma_{0}}<p_{0}$. We find again that $\delta+\gamma_{0}>\pi / 2$, so that the result follows from the second half of Theorem 6.16 in [6].

9. $\Gamma$ meets $C_{\rho_{0}}$ in two reentrant points. If $\Gamma$ is distinct from $\Gamma_{0}$ the only possibility is the configuration of Figure 10; other arcs of a supposed minimizing system are excluded by the above cases. For the original arc $\Gamma_{0}$ we have

$$
\Phi_{0} \text { घ }\left|\Gamma_{0}\right|-\left|\Sigma_{0}^{*}\right| \cos y_{0}+\frac{1}{R_{y_{0}}}\left|\Omega_{0}^{*}\right|=0 \text {. }
$$

Corresponding to the region $\Omega 1 \Omega_{0}^{*}$ we find the corresponding

since $R_{\gamma}=\frac{|\Omega|}{|\Sigma| \cos \gamma}$. There follows

$$
\begin{aligned}
\Phi_{00} & =\left|\Gamma_{0}\right|-|\Sigma| \Sigma_{0}^{*}\left|\cos \gamma_{0}+\frac{1}{R_{\gamma_{0}}}\right| \Omega\left|\Omega_{0}^{*}\right| \\
& =\left|\Gamma_{0}\right|+\left|\Sigma_{0}^{*}\right| \cos \gamma_{0}-\frac{1}{R_{\gamma_{0}}}\left|\Omega_{0}^{*}\right|
\end{aligned}
$$

by (11).

$$
\Phi_{\infty}=2\left|\Gamma_{0}\right|
$$

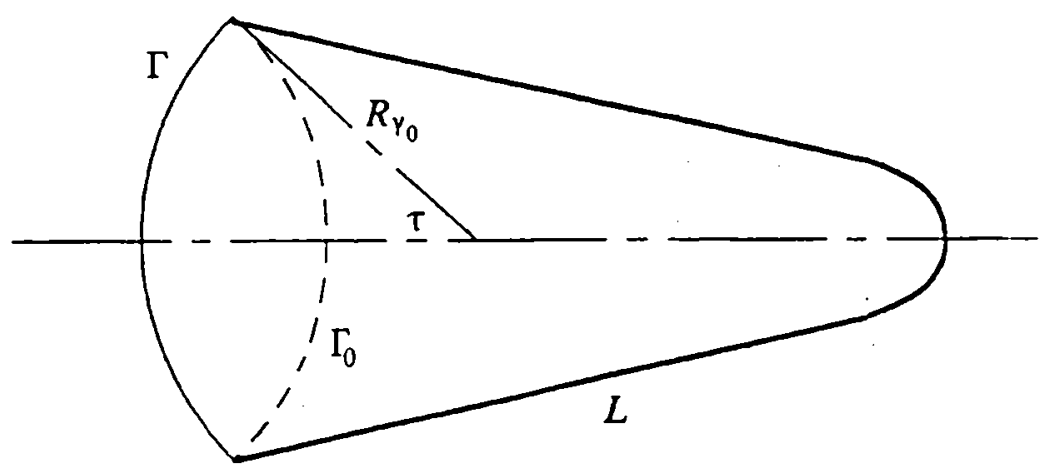

Figure 10. Proof for Case 9

For the curve $\Gamma$ we find

$$
\Phi=\Phi_{00}-\frac{1}{R_{y_{0}}}\left|\Omega_{0}^{* *}\right|=2\left|\Gamma_{\mathrm{d}}\right|-\frac{1}{R_{Y_{0}}}\left|\Omega_{0}^{* *}\right|
$$

where $\Omega_{0}^{* *}$ is the region bounded by $\Gamma$ and $\Gamma_{0}$. We have

$$
\left|\Omega_{0}^{* *}\right|=2 R_{\gamma_{0}}{ }^{2}(\tau-\sin \tau \cos \tau), \quad\left|\Gamma_{0}\right|=2 R_{\gamma_{0}} \tau
$$

Thus

$$
\Phi=2 R_{\gamma_{0}}(\tau+\sin \tau \cos \tau)>0
$$

which contradicts the assumed minimizing property of $\Gamma$. 
We thus conclude that the configuration of Figure 3, when it occurs with $\Phi=0$, is the unique one having this property; a positive solution of the original capillary problem (1) - (4) with finite volume will exist if $\gamma_{0}<\gamma \leq \pi / 2$, but the height of the surface will tend uniformly to infinity over $\Omega_{0}^{*}$ as $\gamma \downarrow \gamma_{0}$.

\section{Stability of the observation}

We wish to examine the response of a given apparatus to changes in the contact angle of the fluid: Specifically, it is important to know that if the particular materials yield a slightly different angle than the one for which the container is built, the size of $\Omega^{*}$ will not decrease dramatically, causing the near discontinuity to be overlooked. We observe that if $\gamma$ changes from $\gamma_{0}$ then $R$ changes according to the relation

$$
R \cos \gamma=R_{0} \cos \gamma_{0} \text {. }
$$

This in turn induces a new "apparent" incident angle $\gamma_{a}$ at the intersection of $\Gamma$ with $L$. Assuming at first $\alpha<\pi / 2$ and that the incident angle at the intersection points is $\gamma_{0}$ (as in the construction), we set $\beta=\pi / 2-\alpha$ and find (see Figure 11)

$$
R \cos \left(\beta+\gamma_{a}\right)=R_{0} \cos \left(\beta+\gamma_{0}\right)
$$

so that

from which

$$
\cos \gamma \cos \left(\beta+\gamma_{0}\right)=\cos \gamma_{0} \cos \left(\beta+\gamma_{a}\right)
$$

$$
0<\frac{\partial \gamma_{a}}{\partial \gamma}=\frac{\cos \left(\beta+\gamma_{0}\right)}{\cos \gamma_{0}} \frac{\sin \gamma}{\sin \left(\beta+\gamma_{a}\right)}<1
$$

at $\gamma=\gamma_{0}=\gamma_{a}$, since $\beta+\gamma_{0}<\pi / 2$.

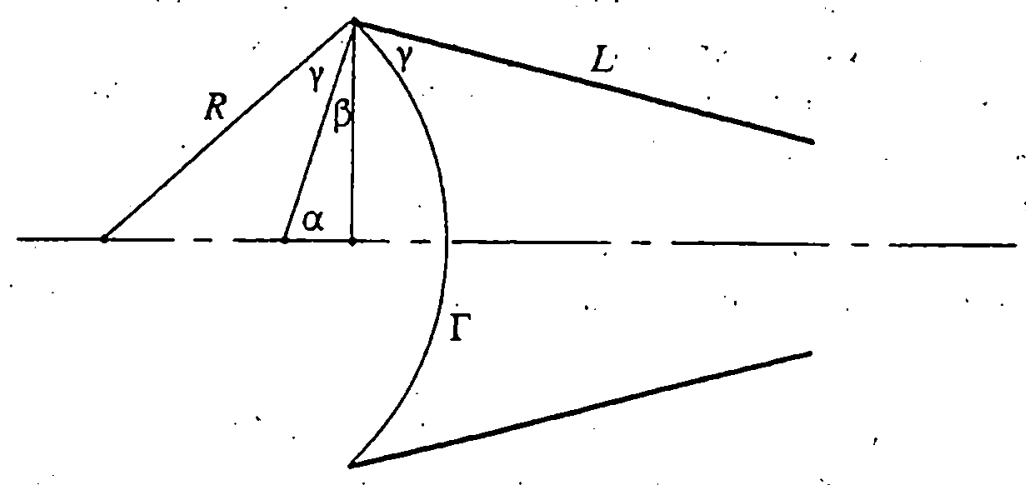

Figure 11: Ohservational stability proof 
If $\gamma>\gamma_{0}$ then $\Phi>0$ and the apparatus will not respond; that is, it will indicate correctly that the angle is too large. If $\gamma<\gamma_{0}$ we conclude from (20) that the apparent angle exceeds $\gamma$ when $\Gamma$ passes through the reentrant points, at least in any range for which (20) continues to hold. But if (20) were to fail there would have to be an initial $\gamma=\gamma^{*}$ at which equality holds on the right side. At this point we have $\gamma^{*}<\gamma_{a}$ so that (20) would hold as stated, a contradiction. Thus as $\gamma$ decreases from $\gamma_{0}$ there continues to hold $\gamma_{a}>\gamma$. But this apparent angle increases when $\Gamma$ is translated along the channel toward $C_{r_{0}}$. It follows that $Y_{a}$ continues to exceed $\gamma$ when $\Gamma$ intersects $L$ at points interior to $L$; that is, $\Gamma$ cannot be extremal in any such configuration, and thus when $\gamma<\gamma_{0}$ the extremal arc $\Gamma$ will continue to pass through the reentrant points, yielding an infinite fluid height in the entire region cut off by $C_{r_{0}}$. Thus there is no danger of missing an angle that is too small due to "disappearance" of the singular set.

If $\alpha=\pi / 2$ then the incident angle $\gamma_{0}$ cannot be realized at the intersection point. That angle must however exceed $\gamma_{0}$, from which one sees easily that an analogous result continues to hold.

\section{Comparisons of critical behavior}

The formal results are summarized in Figures 12, 13, 14, 15. Where appropriate, the corresponding results for the two circle cases studied in $[4]$ are indicated, for comparison purposes, with a dotted line. Figure 12 shows relative size of small radius $r$, extension $h$ and thickness $d$ of the singular portion of the section, as functions of critical angle $\gamma_{0}$, when $\alpha=$ $30^{\circ}$. The lengths are normalized by the maximum horizontal diameter of the section. The crucial curve from the point of view of experiment design is the one for $d$, and one sees that a very large improvement over the two circle case is obtained for small critical angles, an increase of over $50 \%$ being found when $\gamma_{0}=10^{\circ}$. The size of $h$ required for the improvement is remarkably small, being less than $1.5 \%$ of total horizontal diameter. This circumstance indicates that considerable precision in constriction of the apparatus may be needed for accurate results. The requirements do not seem however unreasonable; a change in $h$ of $0.25 \%$ of maximum diameter in the indicated range leads to a change of only about $2^{\circ}$ in measured critical angle.

Corresponding curves for the cases $\alpha=45^{\circ}, 60^{\circ}$, and $90^{\circ}$ are given in Figures 13,14 , 15. These configurations are suited for measurements in successively larger ranges of contact angles. In the case $\alpha=90^{\circ}$, it is not possible to achieve the prescribed critical angle $\gamma_{0}$ between $\Gamma$ and $L$ (unless $\gamma_{0}=0$ ) regardless of the choice of $p$ and $h$. The arbitrary choice $h=$ $r$ was therefore made for this configuration. 

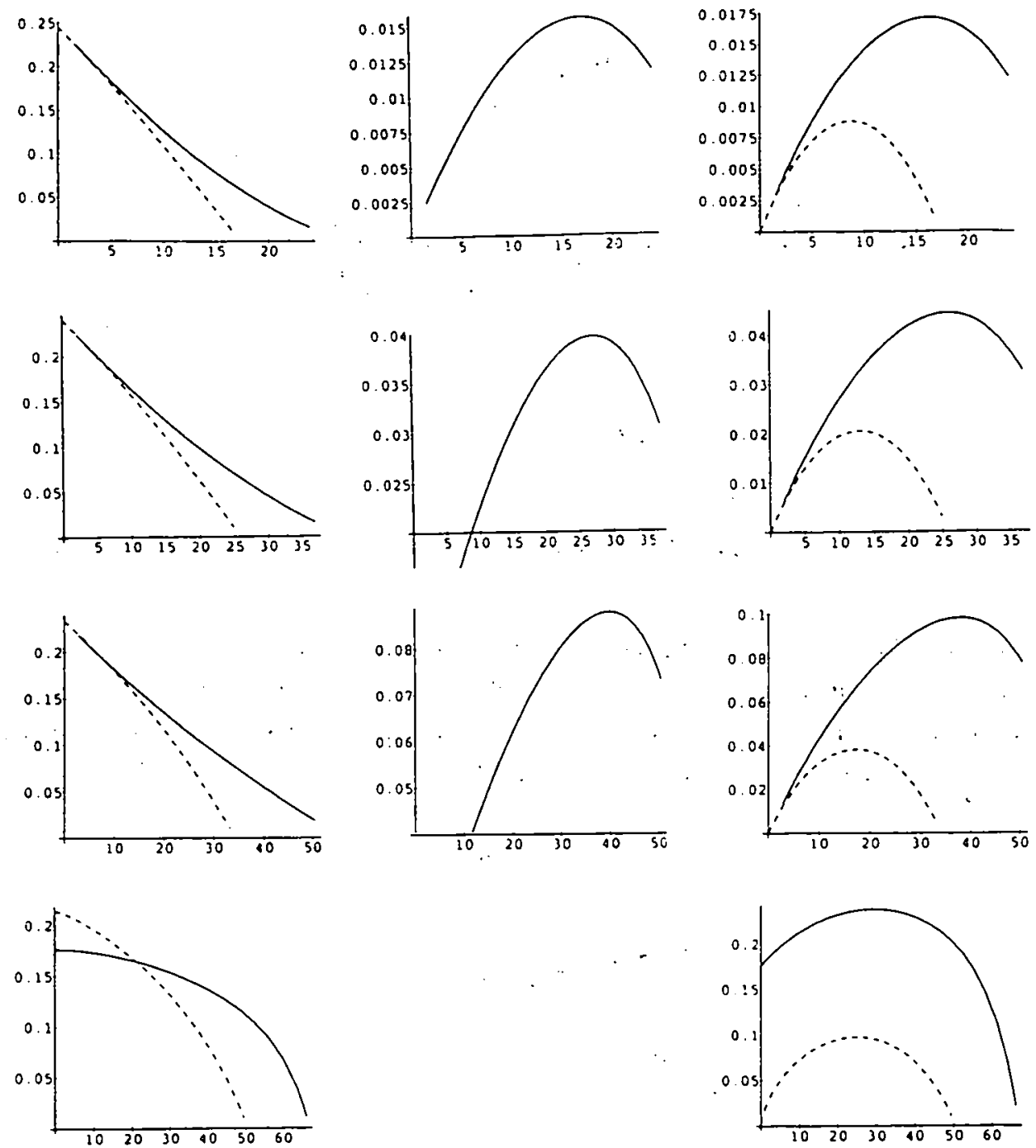

Figures 12-15. Relative sizes of $r, h, d$ as functions of critical angle $\gamma_{0}$; top row, $\alpha=30^{\circ}$; following rows, $\alpha=45^{\circ} .60^{\circ} .90^{\circ}$.

Dashed lines: two circle case. Note varying scales in the figures.

\section{A canonical proboscis}

We show in this section that the container geometry can be so designed as to make the "proboscis" (and the associated thickness $d$ ) as large as desired. We achieve that goal on replacing the segments $L$ and arc $G_{0}$ by arcs admitting at the critical $\gamma_{0}$ an entire continuum of extremals, as 


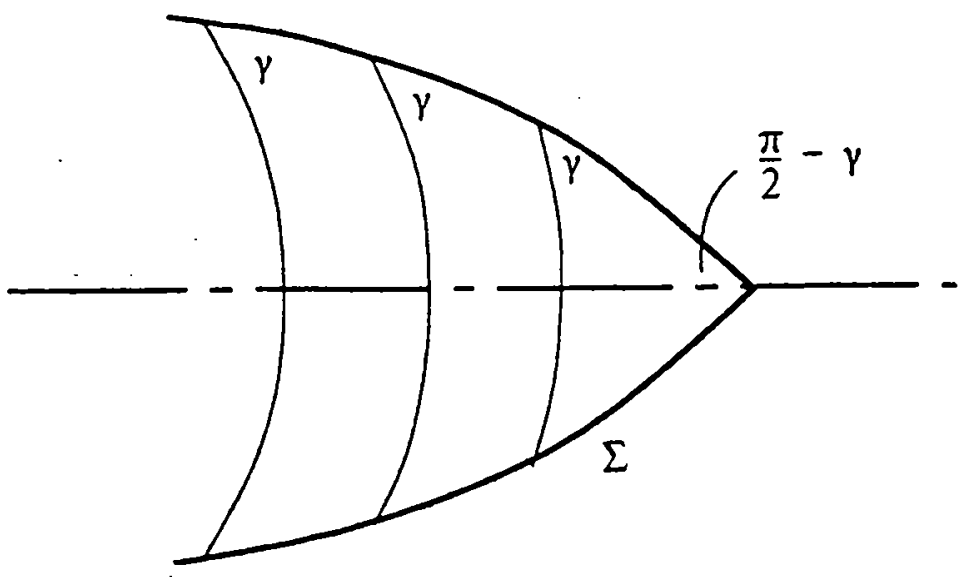

rigure 16. Canonical Proboscis; extremals

indicated in Figure 16. Our initial observation is a necessary condition: if such a continuum is to exist, then since all the radii are equal to the constant $R_{Y_{0}}$, the curve $\Sigma_{0}^{*}$ must have the property that it makes the constant angle $\gamma_{0}$ with a circle of that radius in translatory motion along the symmetry axis. This requirement leads to the equations (see Figure 17)

$$
y=R \cos (\omega+\gamma) . \quad \frac{d y}{d \gamma}=-\tan \omega
$$

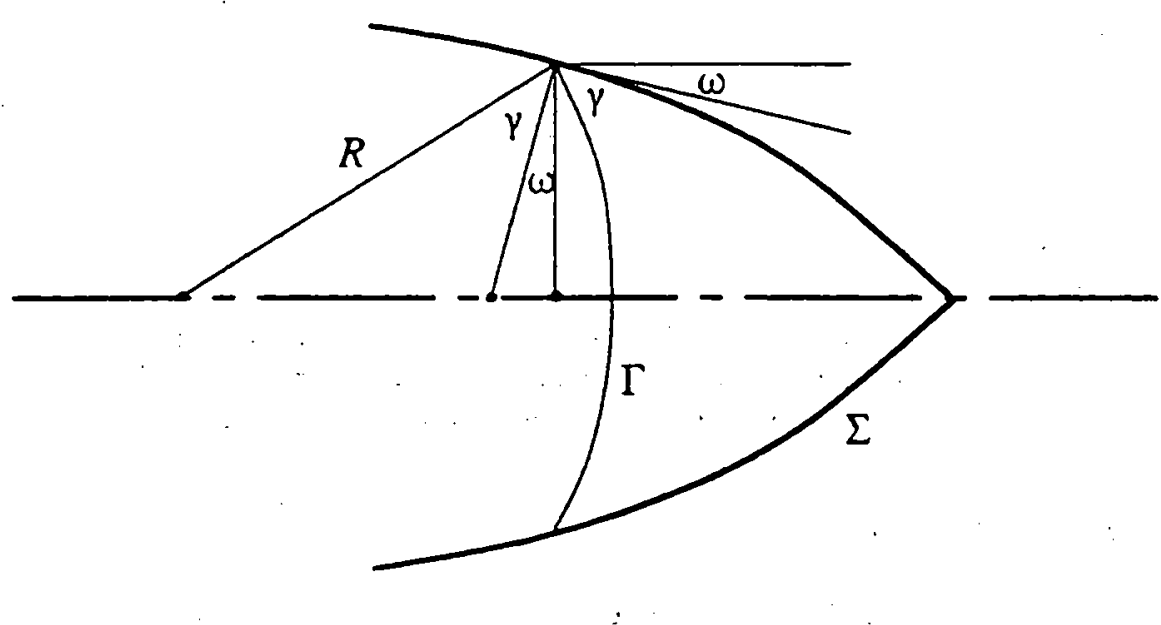

Figure 17. Genesis of equation (21) 
for the upper half of the curves. Here and in what follows, we drop the subscript $\left(_{0}\right)$ in the interest of clarity of the formulas. From (21) we obtain immediately the separable equation

$$
y^{\prime}=\frac{y \sin \gamma-\sqrt{R^{2}-y^{2}} \cos \gamma}{y \cos \gamma+\sqrt{R^{2}-y^{2}} \sin \gamma}
$$

which integrates to

$$
x+c=\sqrt{R^{2}-y^{2}}+R \sin \gamma \ln \frac{\sqrt{R^{2}-y^{2}} \cos \gamma-y \sin \gamma}{R+y \cos \gamma+\sqrt{R^{2}-y^{2}} \sin \gamma} .
$$

A trivial solution of (22) is obtained by setting $y$ equal to the constant value $R \cos \gamma$. The solution family (together with its reflection in the $x$-axis) is sketched in Figure 18. For given $\gamma$, all integral curves between between the lines $y= \pm R \cos \gamma$ are obtained from any given one by rigid horizontal translation; these curves meet the family of all circles, that are enveloped by the two lines, in the constant angle $\gamma$. The curves meet the $x$-axis in the angle $\frac{\pi}{2}-\gamma$, and are asymptotic to the two lines as $x \rightarrow-\infty$.

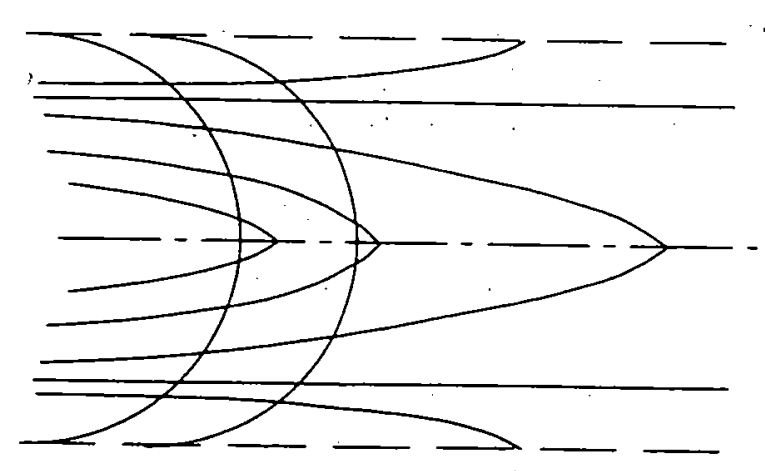

Figure 18. Integral curves of (22); representative extremal arcs

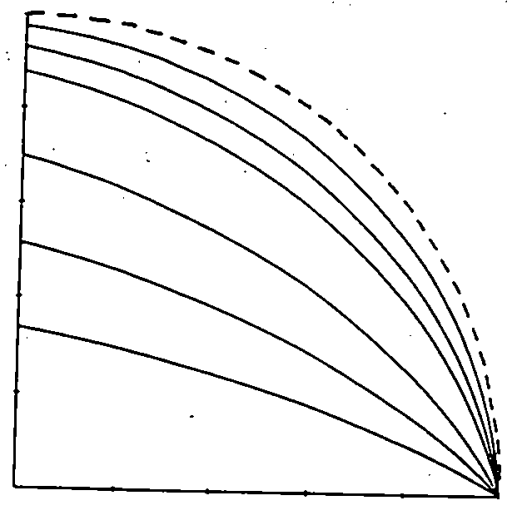

Figure 19. Canonical Proboscises

Figure 19 shows integral curves, corresponding (from the bottom upward) to the angles $\gamma=60,45,30,15,10$, and 5 degrees, and normalized in position so as to coincide on the axis. The dashed curve in the figure is the circular arc that arises when $\gamma=0^{\circ}$. This case is in one sense singular, as the formal integration leads only to the initial circle. However, the two lines $y= \pm R$ now appear as envelopes of the solutions, and can be used to extend any given solution an arbitrary distance to the left of the initial arc. We wish next to complete the 
boundary curve so as to determine a simply connected domain $\Omega$, with the property that $R_{\gamma}=\frac{|\Omega|}{|\Sigma| \cos \gamma}$. This step was effected by introducing a circular arc (Figure 20), the requisite radius $\rho$ being determined

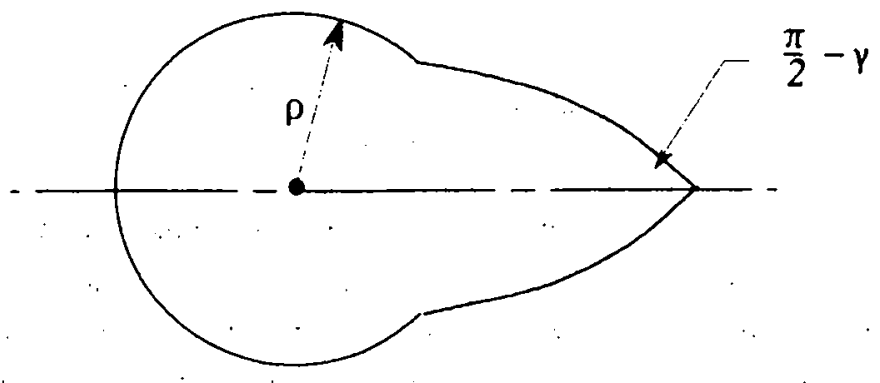

Figure 20. Canonical Section

numerically in cases of particular interest. In all cases tried a solution was possible, and in fact could be achieved with $\rho<2 R$, thus assuring an experimentally feasible configuration. The particular case $\gamma=0^{\circ}$ has here a special interest, as it returns us from a more general point of view to the "keyhole" configuration introduced in $[21$ (see Figure 21); in this case the radius $\rho$ is easily seen to be $(1.974 \ldots) R$, independent of the length $h$ of the proboscis.

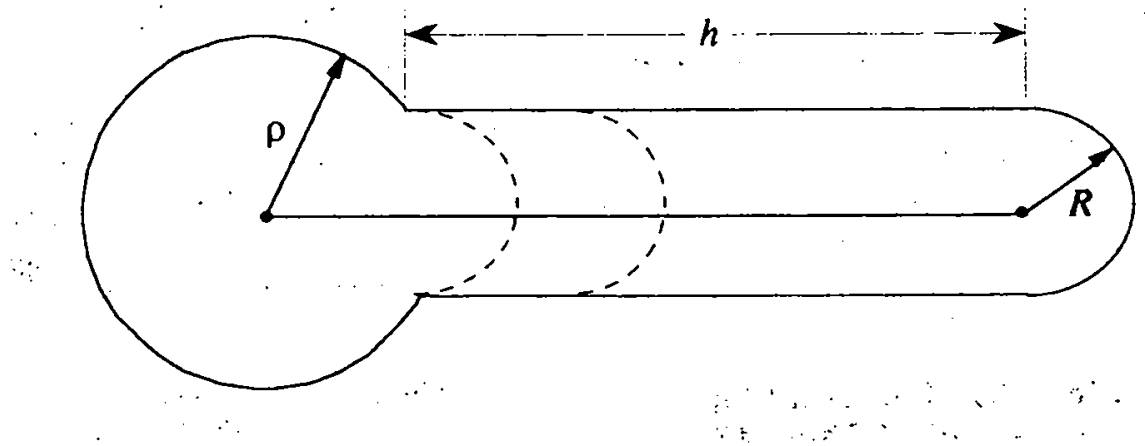

Figure 21. The Keyhole

With the indicated determination of $R$, all the arcs $\Gamma$ become extremals for the subsidiary problem. If we describe them in terms of a parameter $\varepsilon$ by relations $f(x, y ; \varepsilon)=0$, then $\Phi$ becomes a function of $\varepsilon$. Since each $\Gamma$ is extremal, the first variation of $\Phi$ vanishes for any admissible variation. We choose the variation to be $\eta(x, y ; \varepsilon)=\frac{\partial f}{\partial \varepsilon}(x, y ; \varepsilon)$ and obtain immediately that $\dot{\Phi}^{\prime}(\varepsilon)=0$ for every $\varepsilon$, so that $\Phi$ constant for the domains $\Omega$ de termined by the $\operatorname{arcs} \Gamma$. But $\Phi \doteq 0$ at the contact point with the axis, and we conclude that 
$\Phi=0$ for every $\Gamma$ in the family.

The above reasoning determines configurations in which the fluid height can be made to become infinite in a probascis of arbitrarily large length, as the critical angle is crossed. On a tentative basis, we propose these geometries as the canonically best ones for determination of small contact angles. For this purpose, it will be necessary to exclude extraneous extremals as was done in $\$ 2$ above; we intend to return to that point in a later work. Assuming the property, we note that in following the observational stability proof of $\S 3$, when $\alpha<\pi / 2$ the relation (20) now holds on each extremal of the family, and thus when $y$ $<\gamma_{0}$, none of the resulting arcs interior to the proboscis can be extremals. We conclude again that the singular domain cannot disappear discontinuously down the channel with small changes in the contact angle.

We wish to thank Tanya leise for helpful comments.

This investigation was supported in part by a grant from the Natlonal Aeronautics and Space Administration, and in part by grants from the National Science Foundation. The first author is currently supported by a Department of Defense National Defense Science and Engineering Graduate Fellowship.

\section{REFERENCES}

[1] BAINTON, M.C.: Fluid interfaces in the absence of gravity. Report LBL-21665. Berkeley: University of California 1986.:

[2] CONCUS, P. and R. FINN: Continuous and discontinuous disappearance of capillary surfaces. In: Variational Methods for Free Surface Interfaces (eds.: P. Concus and $R$. Finn). New York et al: Springer - Verlag 1987, pp. 197 - 204.

[3] CONCUS, P. and R. FINN: Dichotomous behavior of capillary surfaces in zero gravity. Mikrograv. Sci. and Technol. 3 (1990), 87 - 92.

[4] CONCUS, P. and R. FINN: On accurate determination of contact angle. Proc. IUTAM Symp. Micrograv. Fluid Mech., Bremen 1991 (to appear)..

[5] FINN, R.: A subsidiary variational problem and existence criteria for capillary surfaces. J. Reine Angew. Math. 353 (1984), 196 - 214

[6] FINN, R.: Equilibrium Capillary Surfaces. New York et al: Springer - Vorlag 1986.

[7] FINN, R. : Moon surfaces, and boundary behaviour of capillary surfaces for perfect wetting and non-wotting. Proc. London Math. Soc. 57 (1988), $542-576$.

[8] Miranda, M.: Superfici minime illimitate. Ann. Scuola Norm. Pisa 4 (1977), $313-322$. 